\title{
Evaluating various radiographic methods of shoulder joint damage in patients with rheumatoid arthritis receiving biological disease-modifying antirheumatic drugs
}

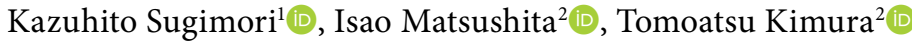 \\ ${ }^{1}$ Department of Orthopaedic Surgery, Toyama Red Cross Hospital, Toyama, Japan \\ ${ }^{2}$ Department of Orthopaedic Surgery, Faculty of Medicine, University of Toyama, Toyama, Japan \\ ${ }^{3}$ Department of Physical and Rehabilitation Medicine, Faculty of Medicine, Kanazawa Medical University, Kahoku, Japan \\ ${ }^{4}$ Shijonawate Gakuen University, Rehabilitation Medicine, Daito, Japan
}

\begin{abstract}
Objectives: This study aims to clarify shoulder joint damage in rheumatoid arthritis patients receiving biological disease-modifying antirheumatic drugs (bDMARDs) and the relationship between joint damage and clinical factors.

Patients and methods: In this retrospective study conducted between April 2005 and December 2008, 36 shoulders in 19 patients (2 males, 17 females; mean age: 58.9 years; range 42 to 75 years) were evaluated at baseline and two years after the initiation of bDMARD therapy with infliximab $(n=14)$ or etanercept $(n=5)$. Standard anteroposterior radiographs of the shoulder joints were taken at baseline and two years after institution of biological therapy. Structural damage in the shoulder joints was assessed using the Larsen scoring method, the medial displacement index (MDI), and the upward migration index (UMI).

Results: There was a significant correlation between MDI, UMI, and Larsen grade before biological therapy. Univariate analysis revealed that the disease activity score 28-count erythrocyte sedimentation rate (ESR) at baseline (odds ratio [OR]: 4.298) was associated with progression of MDI. But multivariate logistic regression revealed that there was no association with the progression of MDI. Univariate analysis revealed that ESR at baseline (OR: 0.967) and matrix metalloproteinase-3 (MMP-3) at baseline (OR: 0.996) were associated with the progression of UMI. Multivariate logistic regression revealed that MMP-3 at baseline (OR: 0.994) was independently associated with the progression of UMI.

Conclusion: Medial displacement index and UMI correlated with the Larsen grade of the shoulder joint strongly and moderately, respectively. This study suggests that MDI and UMI may help to evaluate radiographic progression of damage in shoulder joints in patients on bDMARDs, which is difficult to detect using the Larsen grade.

Keywords: Rheumatoid arthritis, shoulder joint, tumor necrosis factor-alpha.
\end{abstract}

Shoulder joint involvement is often observed in patients with rheumatoid arthritis (RA), and the majority of patients suffer from varying degrees of shoulder symptoms. ${ }^{1}$ In the shoulder joint, the synovial inflammation primarily targets the glenohumeral joint and leads to bony erosions and pain. Such structural damage is common in the shoulder and only a small portion of patients with RA has normal shoulder radiographs throughout the course of their disease. ${ }^{2}$ In affected shoulders with bone and cartilage damage, the subacromial bursa is also involved and the rotator cuff may become trapped between the subacromial bursa and glenohumeral joint is gradually impaired.

\section{Received: May 26, 2020 Accepted: July 16, 2020 Published online: December 12, 2020}

Correspondence: Kazuhito Sugimori, MD. Department of Orthopaedic Surgery, Toyama Red Cross Hospital, $930-8562$ Toyama, Japan. Tel: +81-76-433-2222 e-mail: ksugi0624@gmail.com

Citation:

Sugimori K, Matsushita I, Kimura T. Evaluating various radiographic methods of shoulder joint damage in patients with rheumatoid arthritis receiving biological disease-modifying antirheumatic drugs. Arch Rheumatol 2021;36(3):349-359. 
Range of motion decreases due to pain and structural damage to cartilage, bone and the rotator cuff, causing further functional disability of the shoulder. In the upper extremities, the shoulder joints play a central role in the physical function of $\mathrm{RA}^{3}{ }^{3}$ and, thus, detailed evaluation of the shoulder during medical treatment is indispensable.

Recently, biological agents have shown a major impact on the treatment of RA in controlling disease activity, inhibiting joint destruction, and improving functional status. ${ }^{4}$ Specifically, tumor necrosis factor (TNF)-blocking therapies with biological disease-modifying antirheumatic drugs (bDMARDs) have been shown to inhibit the progression of damage to the small joints in the hands and feet. ${ }^{5}$ Destruction in large joints of the lower extremity, such as hip and knee, is also suppressed by bDMARDs. ${ }^{6,7}$ However, their effects in the glenohumeral joint remain to be clarified. Hirooka et al. ${ }^{8}$ reported that they devised two radiographic parameters: a medial displacement index (MDI) and an upward migration index (UMI) of the humeral head. They studied the natural course and the possibility of making prognoses about shoulder joint destructions in RA patients using these parameters.

The purpose of our study was two-fold. First, it was to evaluate glenohumeral joint damage using several radiographic parameters. We hypothesized that MDI and UMI were useful parameters to detect minor radiographic changes. In addition, it was to analyze factors related to radiographic progression during bDMARD therapy. We hypothesized that there were several factors such as disease activity in association with radiographic progression. Therefore, in this study, we aimed to clarify shoulder joint damage in RA patients receiving bDMARDs and the relationship between joint damage and clinical factors.

\section{PATIENTS AND METHODS}

This study is a retrospective review of consecutive shoulders that received bDMARD therapy between April 2005 and December 2008 in the Faculty of Medicine, University of Toyama. A total of 19 patients (2 males, 17 females; mean age: 58.9 years; 42 to 75 years) were enrolled in this study. All patients fulfilled the
American College of Rheumatology 1987 revised criteria for a diagnosis of RA. ${ }^{9}$ bDMARD therapy was in accordance with the Japan College of Rheumatology Guidelines. ${ }^{10,11}$ Inclusion criteria were active RA with $\geq 6$ swollen joints, $\geq 6$ tender joints, C-reactive protein (CRP) of $\geq 2.0 \mathrm{mg} / \mathrm{dL}$, and an erythrocyte sedimentation rate (ESR) of $\geq 28 \mathrm{~mm} / \mathrm{h}$. All patients had had an inadequate response to one or more recommended levels of conventional DMARDs. Patients were also required to have white blood cell counts of $\geq 4,000 / \mathrm{mm}^{3}$ and peripheral blood lymphocyte counts of $\geq 1,000 / \mathrm{mm}^{3}$. In addition, patients were required to be serum negative for interferon-gamma release assay and $\beta$-D-glucan to avoid possible opportunistic infections, including tuberculosis and Pneumocystis jirovecii pneumonia. Patients were treated with methotrexate (MTX) and a standard dose of infliximab of $3 \mathrm{mg} / \mathrm{kg}$ intravenously at zero, two, and six weeks, and every eight weeks thereafter, or with etanercept at a dose of $25 \mathrm{mg}$ once or twice weekly by subcutaneous injection. The study protocol was approved by the Faculty of Medicine, University of Toyama Ethics Committee (Approval No: 19-11). A written informed consent was obtained from each patient. The study was conducted in accordance with the principles of the Declaration of Helsinki.

Routine laboratory tests, including ESR, CRP, and matrix metalloproteinase-3 (MMP-3), were performed for each patient at baseline and at regular intervals thereafter. As a parameter of disease activity, the Disease Activity Score in 28 joints (DAS28-CRP) ${ }^{12,13}$ was used. Clinical response at one year was defined according to the European League Against Rheumatism (EULAR) response criteria based on the DAS28. ${ }^{14}$ Body mass index that may influence joint damage $e^{15,16}$ was also measured at baseline.

Standard anteroposterior radiographs of the shoulder were taken at baseline and two years after initiation of bDMARD therapy. Joints that had already undergone total joint arthroplasty before the initiation of TNF-blocking therapies were excluded from the radiographic analysis. Structural damage to the joints was assessed by two observers according to Larsen et al. ${ }^{17}$ using standard reference films. In cases of disagreement, a consensus was reached by the observers. The method of Larsen et al. ${ }^{17}$ has reasonable sensitivity and satisfactory intra- and 
inter-observer reliability. ${ }^{18,19}$ The six grades of the Larsen classification are as follows: Grade 0 (no change), the normal status of the joint; Grade I (slight changes), periarticular soft tissue swelling, osteoporosis, and slight joint space narrowing; Grade II (definite early changes), erosion and joint space narrowing correspond to the standards, erosion is obligatory except in the weight-bearing joints; Grade III (medium destructive changes), erosion and joint space narrowing correspond to the standards; Grade IV (severe destructive changes), erosion and joint space narrowing correspond to the standards; and Grade V (mutilating changes), the original articular surfaces have disappeared, gross bone deformation is present.

In addition to the Larsen grade for large joint evaluation, detailed evaluation of joint spaces including the glenohumeral joint and subacromial space were compared for each set of radiographs from each patient as previously described ${ }^{8}$ and as shown in Figure 1. The MDI was obtained by dividing the distance between the center of the humeral head and the glenoid surface $(\mathrm{M})$ by the radius of the humeral head $(\mathrm{R})$. The center of the humeral head was determined using a circle-fitting technique, and then $\mathrm{R}$ was measured. The UMI was obtained by dividing the distance between the center of the humeral head and the central point of the subacromial surface (U) by R. Changes in MDI or UMI were defined as the value resulting from subtracting the post-treatment value from the pre-treatment value.
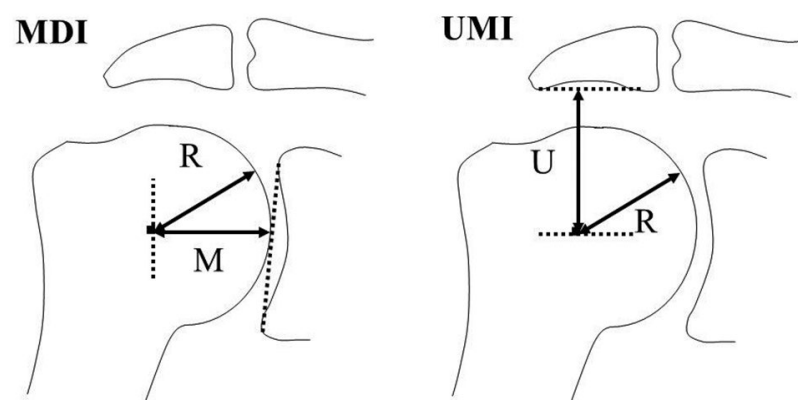

Figure 1. Illustration of measurement methods used in this study.

Left panel: Medial displacement index (MDI); M: Distance between center of humeral head and glenoid surface; R: Radius of humeral head. $\mathrm{MDI}=\mathrm{M} / \mathrm{R}$. Right panel: Upward migration index (UMI); U: Distance between center of humeral head and central point of subacromial surface. $\mathrm{UMI}=\mathrm{U} / \mathrm{R}$.

\section{Statistical analysis}

Wilcoxon's rank sum test was used for continuous variables and the chi-squared test for categorical variables. The frequency of progression of damage in shoulders was compared between Larsen grades and deterioration of MDI and UMI, using Fisher's exact test. Statistical comparison between shoulders with different grades of destruction was performed using analysis of variance. Multivariate logistic analysis was performed to identify the factors associated with the deterioration of MDI or UMI. Variables were considered for the multivariate models if

Table 1. Baseline characteristics of the patients

\begin{tabular}{|c|c|c|c|}
\hline & $\mathrm{n}$ & $\%$ & Mean \pm SD \\
\hline \multicolumn{4}{|l|}{ Demographics variables } \\
\hline Age (year) & & & $58.9 \pm 9.1$ \\
\hline $\begin{array}{l}\text { Sex } \\
\quad \text { Male } \\
\text { Female }\end{array}$ & $\begin{array}{c}2 \\
17\end{array}$ & & \\
\hline \multicolumn{4}{|l|}{ Disease characteristics } \\
\hline Disease duration (year) & & & $15.9 \pm 15.3$ \\
\hline $\begin{array}{c}\text { Stage } \\
\text { I } \\
\text { II } \\
\text { III } \\
\text { IV }\end{array}$ & $\begin{array}{c}1 \\
5 \\
2 \\
11\end{array}$ & & \\
\hline $\begin{array}{c}\text { Class } \\
\text { I } \\
\text { II } \\
\text { III } \\
\text { IV }\end{array}$ & $\begin{array}{c}0 \\
11 \\
8 \\
0\end{array}$ & & \\
\hline $\begin{array}{l}\text { Larsen grade of each shoulder } \\
\text { joint } \\
0 \\
\text { I } \\
\text { II } \\
\text { III } \\
\text { IV } \\
\text { V }\end{array}$ & $\begin{array}{c}13 \\
12 \\
3 \\
5 \\
1 \\
2\end{array}$ & & \\
\hline Body mass index $\left(\mathrm{kg} / \mathrm{m}^{2}\right)$ & & & $22.5 \pm 2.5$ \\
\hline $\mathrm{CRP}(\mathrm{mg} / \mathrm{dL})$ & & & $3.98 \pm 2.4$ \\
\hline $\operatorname{ESR}\left(\mathrm{mm} / 1^{\text {st }} \mathrm{h}\right)$ & & & $78.7 \pm 24.4$ \\
\hline MMP-3 (ng/mL) & & & $319.2 \pm 206.6$ \\
\hline DAS28-ESR & & & $5.83 \pm 0.76$ \\
\hline \multicolumn{4}{|l|}{ Concomitant treatment } \\
\hline Concomitant methotrexate & 17 & 89.5 & \\
\hline Methotrexate dose (mg/week) & & & $6.16 \pm 2.6$ \\
\hline Concomitant corticosteroids & 15 & 78.9 & \\
\hline Corticosteroid dose (mg/day) & & & $3.33 \pm 2.4$ \\
\hline
\end{tabular}

Except where indicated otherwise, values are the median (interquartile range). SD: Standard deviation; CRP: C-reactive protein; ESR: Erythrocyte sedimentation rate; MMP-3: Matrix metalloproteinase-3; DAS28: Disease Activity Score 28-joint assessment; MTX: Methotrexate. 
Table 2. Radiographic assessment according to Larsen grade, MDI and UMI at baseline

\begin{tabular}{|c|c|c|c|c|}
\hline & \multicolumn{2}{|c|}{ Number of shoulders before TNF-blocking therapies } & \multirow{2}{*}{$\begin{array}{c}\text { MDI (pre) } \\
\text { Mean } \pm \text { SD }\end{array}$} & \multirow{2}{*}{$\begin{array}{c}\text { UMI (pre) } \\
\text { Mean } \pm \text { SD }\end{array}$} \\
\hline & $\mathrm{n}$ & $\%$ & & \\
\hline \multicolumn{5}{|l|}{ Larsen grade } \\
\hline 0 & 13 & 36.1 & $1.007 \pm 0.069$ & $1.375 \pm 0.108$ \\
\hline I & 12 & 33.3 & $1.001 \pm 0.019$ & $1.421 \pm 0.067$ \\
\hline II & 3 & 8.3 & $0.871 \pm 0.027$ & $1.309 \pm 0.069$ \\
\hline III & 5 & 13.9 & $0.865 \pm 0.038$ & $1.314 \pm 0.046$ \\
\hline IV & 1 & 2.8 & 0.682 & 1.045 \\
\hline $\mathrm{V}$ & 2 & 5.6 & $0.646 \pm 0.105$ & $1.111 \pm 0.072$ \\
\hline Total & 36 & 100 & $0.945 \pm 0.121$ & $1.353 \pm 0.131$ \\
\hline ANOVA* ( $p$ value) & & & $<0.0001$ & 0.0012 \\
\hline
\end{tabular}

their univariate $p$ value was $<0.05$ and two or three variables of fewer $p$ values, and odds ratios (ORs) and 95\% confidence intervals (CIs) were calculated. Values of $p<0.05$ were considered statistically significant. Post hoc power analysis for comparing deterioration of MDI and UMI and no deterioration of MDI and UMI were performed. A minimum sample size recruited into each arm was calculated to detect deterioration of MDI and UMI, with type-I (alpha) error set at 0.05 and type-II (beta) error set at 0.2 (80\% power). Standard deviation of MDI and UMI were 0.067 and 0.0977 , respectively. The mean difference of deterioration and no deterioration of MDI and UMI were 0.1403 and 0.163 , respectively. The calculated effect size of MDI and UMI were 10 and 14, respectively. All analyses were performed using JMP for Windows, version 14.2 (SAS Institute Inc., Cary, NC, USA).

\section{RESULTS}

Table 1 shows the baseline characteristics of patients. Most patients (89.5\%) received MTX before bDMARD therapy either as (a)

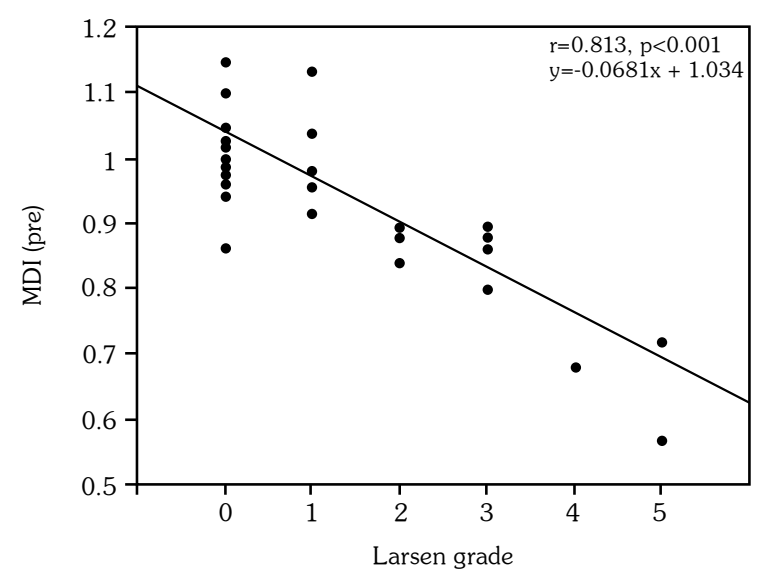

(b)

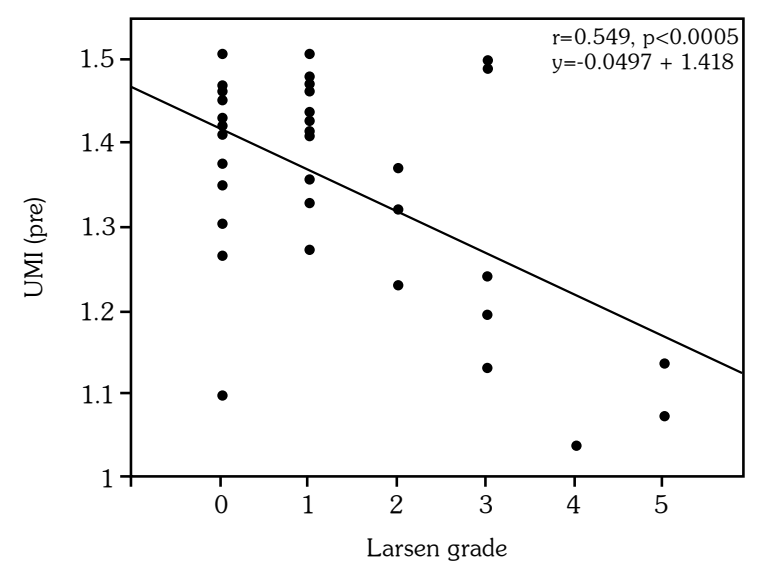

Figure 2. (a) Correlation between medial displacement index and Larsen grade pre-biological disease-modifying antirheumatic drug therapy. There was a significant correlation between medial displacement index and Larsen grade $(\mathrm{r}=0.813, \mathrm{p}<0.001)$. (b) Correlation between upward migration index and Larsen grade pre-biological disease-modifying antirheumatic drug therapy. There was a significant correlation between upward migration index and Larsen grade $(\mathrm{r}=0.549, \mathrm{p}=0.0005)$.

MDI: Medial displacement index; UMI: Upward migration index. 
monotherapy or in combination with different conventional DMARDs. A total of 15 patients (78.9\%) received corticosteroids, with a mean dose of 3.33 (interquartile range, 2-7) mg/day. Patients had moderate $(n=16)$ or high $(n=3)$ disease activity. Infliximab $(n=14)$ and etanercept $(n=5)$ were administered to patients (including cases that switched from infliximab). A total of 36 glenohumeral joints, excluding joints with preceding surgery, were analyzed for their baseline Larsen grades as follows: Grade 0, 13 joints (36.1\%); Grade I, 12 joints (33.3\%); Grade II, three joints (8.3\%); Grade III, five joints (13.9\%); Grade IV, one joint (2.8\%); and Grade V, two joints (5.6\%).

We compared the values of MDI and UMI among the Larsen grades. Mean values of MDI and UMI were both significantly related to increased Larsen grades pre-bDMARD therapy (Table 2). There was a strong correlation between MDI and Larsen grade at baseline (correlation coefficient: $r=0.813, \quad p<0.001$ ) (Figure 2a). Similarly, there was a moderate correlation between UMI and Larsen grade (correlation coefficient: $\mathrm{r}=0.549, \mathrm{p}=0.0005$ ) (Figure 2b). Therefore, both MDI and UMI might be useful markers of radiographic damage similar to the Larsen grading system.

Figure $2 \mathrm{a}$ and $2 \mathrm{~b}$ show the tilt of generalized linear model. For each increase in Larsen grade, MDI decreased by 0.0681 and UMI decreased by 0.0497. In this study, we defined that deterioration of MDI and UMI would result in a negative number after the subtraction of the post-treatment MDI and UMI values from the pre-treatment MDI and UMI values, respectively.

Assessment of radiographs of the 36 glenohumeral joints indicated Larsen grade progression in nine joints (25\%) (two joints from Grades 0 to I, one joint of Grade 0 to II, one joint of Grade II to III, three joints of Grade III to $\mathrm{IV}$, one joint of Grade III to $\mathrm{V}$, and one joint of Grade IV to V) (Figure 3a).

Next, we compared MDI and UMI changes pre- and post-bDMARD therapy (Figure $3 \mathrm{~b}$ and $3 \mathrm{c}$ ). There was no significant difference between pre- and post-treatment MDI values $(p=0.451)$. Similarly, there was no significant difference between pre- and posttreatment UMI values $(p=0.835)$. (a)

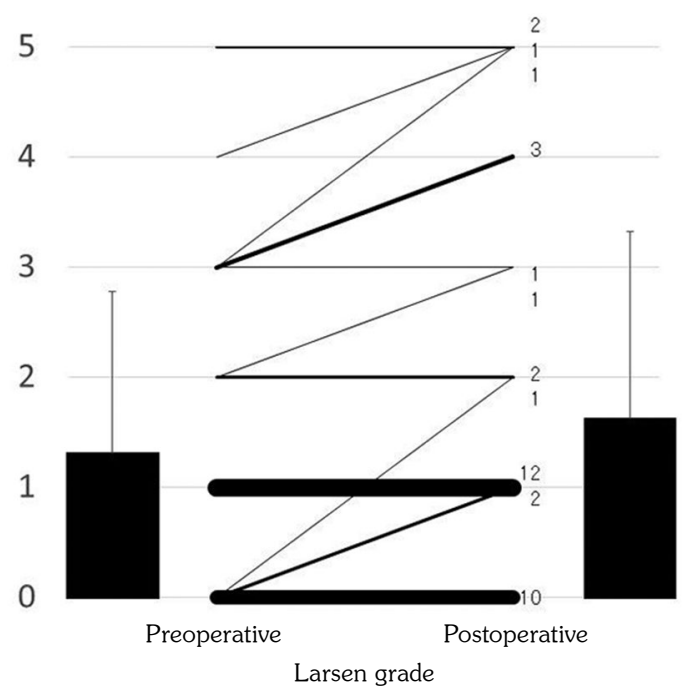

(b)

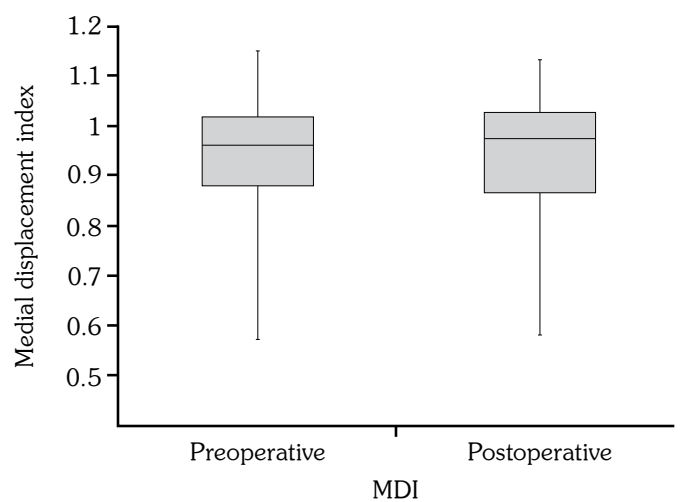

(c)

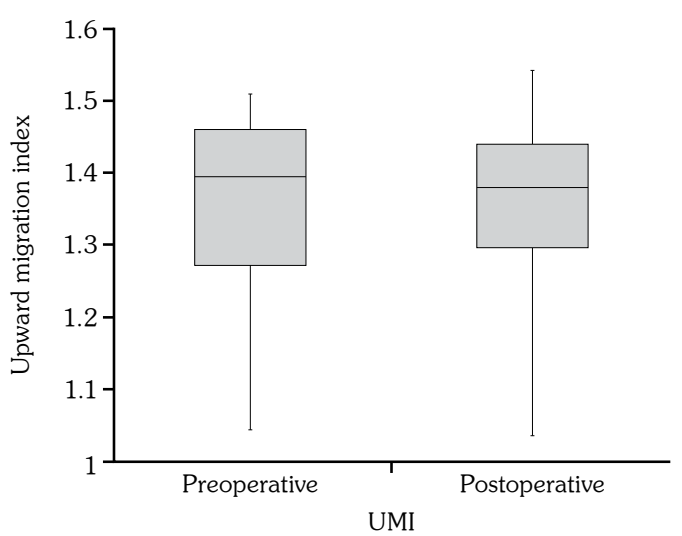

Figure 3. (a) Comparison of Larsen grade of preand post-biological disease-modifying antirheumatic drug therapy. Line graph presenting Larsen grade of each shoulder. Numbers on right side are shoulders at each grade. Bar graph presents mean and standard deviation of Larsen grade. (b) Box plot for medial displacement index changes between pre- and post-treatment. (c) Box plot for upward migration index changes between pre- and posttreatment.

MDI: Medial displacement index; UMI: Upward migration index. 


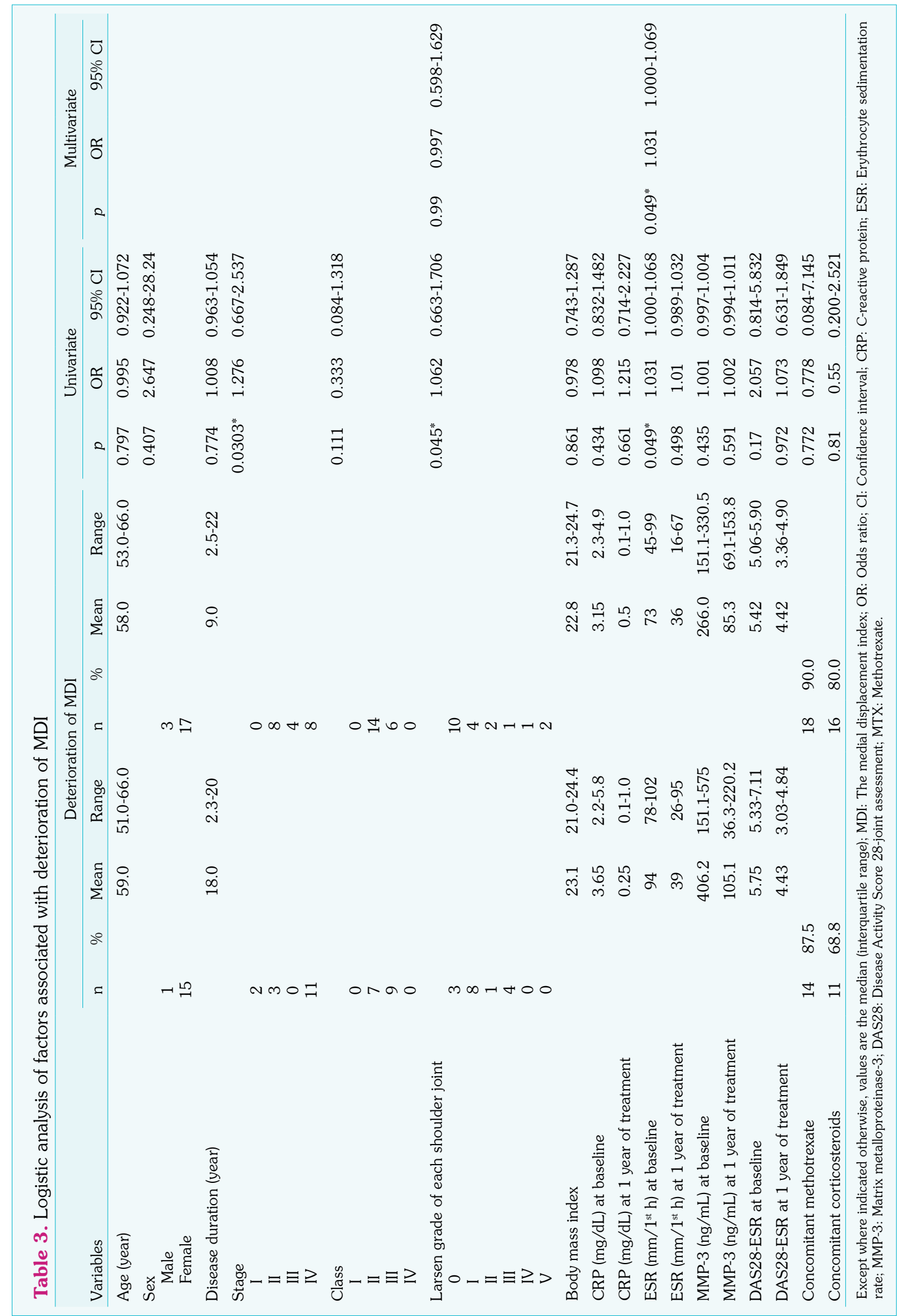




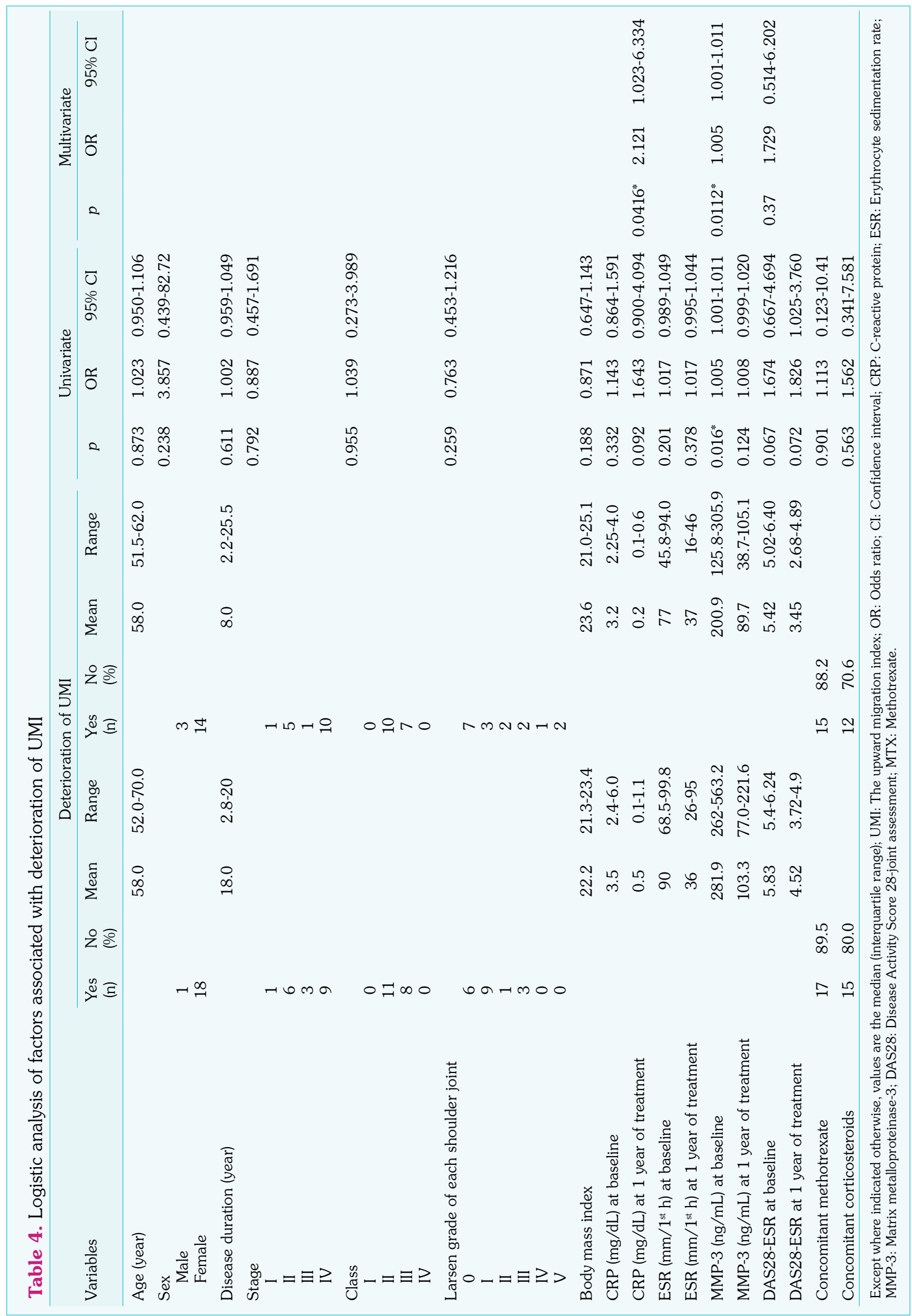


We performed logistic regression analysis to reveal the factors associated with the progression of joint space narrowing, which was given signs of MDI and UMI.

Univariate analysis revealed that Steinbrocker stage (OR: 1.276, 95\% CI: 0.667-2.537), Larsen grade at baseline (OR: 1.062, 95\% CI: 0.6631.706), and ESR at baseline (OR: 1.031, 95\% CI: 1.000-1.068) were associated with the deterioration of MDI (Table 3). Before multivariate analysis, we checked multicollinearity among these factors using Spearman's rank correlation coefficient. This analysis showed significant correlations between Steinbrocker stage and Larsen grade ( $p=0.003, r=0.488)$. We excluded Steinbrocker stage and performed multivariate analysis with Larsen grade and ESR at baseline. Multivariate logistic regression revealed that ESR at baseline was independently associated with the deterioration of MDI (OR: 1.031, 95\% CI: 1.000-1.069).

Univariate analysis revealed that MMP-3 at baseline (OR: 1.005, 95\% CI: 1.001-1.011) was associated with the deterioration of UMI (Table 4). Next, we included CRP at one year of treatment, MMP-3 at one year of treatment, DAS28-ESR at baseline, and DAS28-ESR at one year of treatment in multivariate analysis. Before multivariate analysis, we checked multicollinearity among these factors using Spearman's rank correlation coefficient. This analysis showed the significant correlations between MMP-3 at one year of treatment and DAS28-ESR at one year of treatment $(p=0.015$, $r=0.425)$, MMP-3 at one year of treatment and DAS28-ESR at baseline $(p=0.027, r=0.392)$, MMP-3 at one year of treatment and DAS28-ESR at one year of treatment $(p=0.015, r=0.425)$, CRP at one year of treatment and DAS28-ESR at one year of treatment $(p<0.001, r=0.670)$. We excluded MMP-3 at one year of treatment and DAS28-ESR at one year of treatment and performed multivariate analysis with CRP at one year of treatment, MMP-3 at baseline, and DAS28-ESR at baseline. Multivariate logistic regression revealed that $\mathrm{CRP}$ at one year of treatment (OR: 2.121, 95\% CI: 1.023-6.334) and MMP-3 at baseline (OR: 1.005, 95\% CI: 1.001-1.011) were independently associated with the deterioration of UMI.
Receiver operating characteristic (ROC) curves for ESR at baseline and the deterioration of MDI were constructed. The area under the ROC curve (AUC) was 0.698 and cut-off ESR at baseline was 78.0. The sensitivity and specificity of the ESR at baseline were $86.7 \%$ and $63.2 \%$, respectively. OR for the deterioration of MDI was 1.031.

The ROC curves for CRP at one year of treatment and MMP-3 at baseline and the deterioration of UMI were constructed. The AUC was 0.661 and cut-off CRP at one year of treatment was 0.400 . The sensitivity and specificity of the CRP at one year of treatment were $68.4 \%$ and $70.6 \%$, respectively. OR for the deterioration of UMI was 1.643. The AUC was 0.743 and cut-off MM-3 at baseline was 266.0. The sensitivity and specificity of the MMP-3 data were $72.2 \%$ and $68.7 \%$, respectively. OR for the deterioration of UMI was 1.005 .

\section{DISCUSSION}

A report from the Acute Venous Thrombosis: Thrombus Removal With Adjunctive CatheterDirected Thrombolysis (ATTRACT) study showed that increases of modified total Sharp scores were inhibited by infliximab administration in comparison with MTX alone. Approximately $50 \%$ of patients receiving infliximab showed radiological improvement. ${ }^{5}$ Previously, Seki et al. $^{6}$ reported that bDMARD therapy could inhibit the progression of weight-bearing joint damage as well as in small joints. However, hip and knee joints with Larsen Grade III or IV damage at baseline showed progression even in patients with a good response to the bDMARD treatment. In the previous work of Matsushita et al., ${ }^{7}$ it was also demonstrated that independent factors associated with progression of damage in the hip and knee were baseline Larsen grade and disease activity at one year after bDMARD therapy. There have been few reports regarding the effects of bDMARD therapy on shoulder joints. In the present study, we observed a similar therapeutic effect on the shoulder joints of RA patients receiving bDMARDs. There were few damaged joints of baseline Larsen Grades 0-II and there was a low rate of progression of Larsen grade after bDMARD therapy was initiated. Therefore, shoulder 
joints responded similarly to weight-bearing joints after bDMARD therapy. Thus, it is expected that the progression of shoulder joint destruction can be controlled when damage of the joint is scored as Larsen Graderade 0-II.

Although the most widely used evaluation of the large joints of RA is the Larsen grade, it is not specifically designed for rheumatoid shoulder lesions, and it is often difficult to differentiate among Larsen Grades III-V. Several reports have described the use of the acromiohumeral interval and glenohumeral joint space as an index of deviations of the humeral head. ${ }^{20-22}$ However, shoulders are difficult to assess, particularly when severe destruction is present and they can be easily affected by the photographic method such as radiographic amplification and gaps in the photographic direction. Therefore, we also used two indices, MDI and UMI, reported by Hirooka et al., ${ }^{8}$ to evaluate the shoulder joints of RA patients. Similar to Hirooka et al.'s ${ }^{8}$ report, our results suggested that MDI and UMI were useful parameters for quantitatively assessing the radiographic progression of shoulder joints in RA patients receiving bDMARD therapy.

Univariate analysis demonstrated that stage, Larsen grade, and ESR at baseline were higher in patients with MDI deterioration pre-treatment. Moreover, ESR at baseline was correlated with the deterioration of MDI at multivariate analysis. CRP at one year of treatment and MMP-3 at baseline were higher in the patients with pre-treatment UMI deterioration. On the other hand, MMP-3 might have statistical significance in UMI at preand post-bDMARD therapy. MMP-3 is known to be a major cartilage-degrading enzyme, which is produced by synovial lining cells and chondrocytes themselves. ${ }^{23,24}$ It is reported that levels of MMP-1 and MMP-3 in knee synovial fluid were significantly higher in RA than in osteroarthrits. ${ }^{25}$ Yoshihara et al. ${ }^{26}$ reported that levels of MMP-1, MMP-3, tissue inhibitor of metalloproteinase-1, and glycosaminoglycan in synovial fluid from patients with full-thickness tears of the rotator cuff appeared to be higher than those with partialthickness tears. Therefore, MMP-3 might be a significant factor influencing UMI deterioration that might be affected by a rotator cuff tear. In the previous work of Matsushita et al., ${ }^{7}$ it was reported that the progression of damage in hip and knee joints was influenced by baseline Larsen grade.
On the other hand, baseline Larsen grade was not affected by the deterioration of MDI and UMI in multivariate analysis of non-weight-bearing joints. We speculate that the shoulder joint is less susceptible to mechanical stress.

In our results, radiographic progression of shoulder joints was 9 of 36 (25\%) by Larsen grade. But in detail, there was no significant difference between pre- and post-treatment MDI values. Moreover, there was no significant difference between pre- and post-treatment UMI values. Matsushita et al. ${ }^{7}$ reported that radiographic evidence of damage progression by Larsen grade was present in 11.5\%-15.9\% of hip, knee, ankle, and subtalar joints at one-three years after bDMARDs therapy. There might be some difference of effectiveness of joint damage between shoulder joints and weight-bearing joints after bDMARDs therapy.

Lehtinen et al. ${ }^{27}$ reported that upper migration of the humeral head slightly precedes medial migration. In addition, the upward migration of the humeral head might rapidly progress when it is accompanied by a rotator cuff tear due to inflammation, even in the presence of minimal joint destruction. ${ }^{28}$ Weiner and Macnab $^{20}$ examined 59 shoulders with surgically detected rotator cuff tears and found that half of these patients had a subacromial space $<6 \mathrm{~mm}$, compared with no values $<7 \mathrm{~mm}$ in 60 normal shoulders. In this study, we observed subacromial space narrowing $(<7 \mathrm{~mm})$ in seven shoulders. Moreover, there were only two shoulders with $6 \mathrm{~mm}$ of subacromial space in joints of Larsen Grade 0-II. We should consider the presence of a rotator cuff tear if progression of UMI is observed without good clinical response as assessed by the EULAR criteria. According to our univariate analysis, DAS28-ESR at baseline and one year of treatment was not significantly different between deterioration and no deterioration of MDI and UMI.

The present study has several limitations. First, the current results were obtained from a small number of patients treated with two types of bDMARDs at a single center. Moreover, a small number of joints with deterioration may decrease the reliability of the results of the multivariate analysis. Second, we did not analyze radiographic changes of shoulder joints in patients without 
bDMARD therapy. Nevertheless, the data from this study should be useful for understanding the effects and limits of bDMARD therapy on shoulder joints with different degrees of baseline radiographic damage. In the future, the relationship of the change of MDI and UMI and the deterioration of shoulder destruction should be revealed and the difference of MDI and UMI according to shoulder destruction pattern.

In conclusion, both MDI and UMI were strongly and moderately correlated with Larsen grade of the shoulder joint, respectively. Our results showed that the parameters of MDI and UMI can be easily used to detect minor radiographic changes, which are hard to detect using the Larsen grade. Multivariate logistic regression revealed that ESR at baseline was independently associated with the progression of MDI. Multivariate logistic regression revealed that MMP-3 at baseline and $\mathrm{CRP}$ at one year of treatment were independently associated with the progression of UMI.

\section{Declaration of conflicting interests}

The authors declared no conflicts of interest with respect to the authorship and/or publication of this article.

\section{Funding}

The authors received no financial support for the research and/or authorship of this article.

\section{REFERENCES}

1. Cuomo F, Greller MJ, Zuckerman JD. The rheumatoid shoulder. Rheum Dis Clin North Am 1998;24:67-82.

2. Thomas T, Noël E, Goupille P, Duquesnoy B, Combe B; GREP. The rheumatoid shoulder: current consensus on diagnosis and treatment. Joint Bone Spine 2006;73:139-43.

3. Shidara K, Inoue E, Hoshi D, Tanaka E, Seto Y, Nakajima $\mathrm{A}$, et al. The influence of individual joint impairment on functional disability in rheumatoid arthritis using a large observational database of Japanese patients. J Rheumatol 2012;39:476-80.

4. Bathon JM, Martin RW, Fleischmann RM, Tesser JR, Schiff MH, Keystone EC, et al. A comparison of etanercept and methotrexate in patients with early rheumatoid arthritis. N Engl J Med 2000;343:1586-93.

5. Lipsky PE, van der Heijde DM, St Clair EW, Furst DE, Breedveld FC, Kalden JR, et al. Infliximab and methotrexate in the treatment of rheumatoid arthritis. Anti-Tumor Necrosis Factor Trial in Rheumatoid
Arthritis with Concomitant Therapy Study Group. N Engl J Med 2000;343:1594-602.

6. Seki E, Matsushita I, Sugiyama E, Taki H, Shinoda $\mathrm{K}$, Hounoki $\mathrm{H}$, et al. Radiographic progression in weight-bearing joints of patients with rheumatoid arthritis after TNF-blocking therapies. Clin Rheumatol 2009;28:453-60.

7. Matsushita I, Motomura H, Seki E, Kimura T. Radiographic changes and factors associated with subsequent progression of damage in weight-bearing joints of patients with rheumatoid arthritis under TNFblocking therapies-three-year observational study. Mod Rheumatol 2017;27:570-5.

8. Hirooka A, Wakitani S, Yoneda M, Ochi T. Shoulder destruction in rheumatoid arthritis. Classification and prognostic signs in 83 patients followed 5-23 years. Acta Orthop Scand 1996;67:258-63.

9. Arnett FC, Edworthy SM, Bloch DA, McShane DJ, Fries JF, Cooper NS, et al. The American Rheumatism Association 1987 revised criteria for the classification of rheumatoid arthritis. Arthritis Rheum 1988;31:315-24.

10. Miyasaka N, Takeuchi T, Eguchi K. Proposed [corrected] Japanese guidelines for the use of infliximab for rheumatoid arthritis. Mod Rheumatol 2005;15:4-8.

11. Miyasaka N, Takeuchi T, Eguchi K. Guidelines for the proper use of etanercept in Japan. Mod Rheumatol 2006;16:63-7.

12. van der Heijde DM, van 't Hof MA, van Riel PL, Theunisse LA, Lubberts EW, van Leeuwen MA, et al. Judging disease activity in clinical practice in rheumatoid arthritis: first step in the development of a disease activity score. Ann Rheum Dis 1990;49:91620.

13. Prevoo ML, van 't Hof MA, Kuper HH, van Leeuwen MA, van de Putte LB, van Riel PL. Modified disease activity scores that include twenty-eight-joint counts. Development and validation in a prospective longitudinal study of patients with rheumatoid arthritis. Arthritis Rheum 1995;38:44-8.

14. van Gestel AM, Prevoo ML, van 't Hof MA, van Rijswijk $\mathrm{MH}$, van de Putte LB, van Riel PL. Development and validation of the European League Against Rheumatism response criteria for rheumatoid arthritis. Comparison with the preliminary American College of Rheumatology and the World Health Organization/ International League Against Rheumatism Criteria. Arthritis Rheum 1996;39:34-40.

15. Kaufmann J, Kielstein V, Kilian S, Stein G, Hein G. Relation between body mass index and radiological progression in patients with rheumatoid arthritis. $J$ Rheumatol 2003;30:2350-5.

16. van der Helm-van Mil AH, van der Kooij SM, Allaart CF, Toes RE, Huizinga TW. A high body mass index has a protective effect on the amount of joint destruction in small joints in early rheumatoid arthritis. Ann Rheum Dis 2008;67:769-74. 
17. Larsen A, Dale K, Eek M. Radiographic evaluation of rheumatoid arthritis and related conditions by standard reference films. Acta Radiol Diagn (Stockh) 1977; 18:481-91.

18. Guth A, Coste J, Chagnon S, Lacombe P, Paolaggi JB. Reliability of three methods of radiologic assessment in patients with rheumatoid arthritis. Invest Radiol 1995;30:181-5.

19. Scott DL, Laasonen L, Priolo F, Houssien DA, Bacarini $\mathrm{L}$, Cerase A, et al. The radiological assessment of rheumatoid arthritis. Clin Exp Rheumatol 1997;15 Suppl 17:S53-61.

20. Weiner DS, Macnab I. Superior migration of the humeral head. A radiological aid in the diagnosis of tears of the rotator cuff. $\mathrm{J}$ Bone Joint Surg $[\mathrm{Br}]$ 1970;52:524-7.

21. Crossan JF, Vallance R. The shoulder joint in rheumatoid arthritis. In: Barley I, Kessel L, editors. Shoulder Surgery. Berlin: Springer Verlag; 1982. p. 131-9.

22. Petersson CJ, Redlund-Johnell I. The subacromial space in normal shoulder radiographs. Acta Orthop Scand 1984;55:57-8.

23. Freemont AJ, Hampson V, Tilman R, Goupille P, Taiwo Y, Hoyland JA. Gene expression of matrix metalloproteinases 1,3 , and 9 by chondrocytes in osteoarthritic human knee articular cartilage is zone and grade specific. Ann Rheum Dis 1997;56:542-9.

24. Okada Y, Takeuchi N, Tomita K, Nakanishi I, Nagase $\mathrm{H}$. Immunolocalization of matrix metalloproteinase 3 (stromelysin) in rheumatoid synovioblasts (B cells): correlation with rheumatoid arthritis. Ann Rheum Dis 1989;48:645-53.

25. Yoshihara Y, Nakamura H, Obata K, Yamada H, Hayakawa T, FujikawaK, etal. Matrixmetalloproteinases and tissue inhibitors of metalloproteinases in synovial fluids from patients with rheumatoid arthritis or osteoarthritis. Ann Rheum Dis 2000;59:455-61.

26. Yoshihara Y, Hamada K, Nakajima T, Fujikawa K, Fukuda H. Biochemical markers in the synovial fluid of glenohumeral joints from patients with rotator cuff tear. J Orthop Res 2001;19:573-9.

27. Lehtinen JT, Belt EA, Kauppi MJ, Kaarela K, Kuusela PP, Kautiainen HJ, et al. Bone destruction, upward migration, and medialisation of rheumatoid shoulder: a 15 year follow up study. Ann Rheum Dis 2001;60:322-6.

28. Lehtinen JT, Belt EA, Lybäck CO, Kauppi MJ, Kaarela K, Kautiainen HJ, et al. Subacromial space in the rheumatoid shoulder: a radiographic 15-year follow-up study of 148 shoulders. J Shoulder Elbow Surg 2000;9:183-7. 